\title{
Age-Dependent Increase in Incidence of Staphylococcus aureus Bacteremia, Denmark, 2008-2015
}

\author{
Louise Thorlacius-Ussing, Haakon Sandholdt, Anders Rhod Larsen, \\ Andreas Petersen, Thomas Benfield
}

\section{Medscape ACTIVITY}

In support of improving patient care, this activity has been planned and implemented by Medscape, LLC and Emerging Infectious Diseases. Medscape, LLC is jointly accredited by the Accreditation Council for Continuing Medical Education (ACCME), the Accreditation Council for Pharmacy Education (ACPE), and the American Nurses Credentialing Center (ANCC), to provide continuing education for the healthcare team.

Medscape, LLC designates this Journal-based CME activity for a maximum of 1.00 AMA PRA Category 1 Credit(s) ${ }^{\mathrm{TM}}$. Physicians should claim only the credit commensurate with the extent of their participation in the activity.

Successful completion of this CME activity, which includes participation in the evaluation component, enables the participant to earn up to $1.0 \mathrm{MOC}$ points in the American Board of Internal Medicine's (ABIM) Maintenance of Certification (MOC) program. Participants will earn MOC points equivalent to the amount of CME credits claimed for the activity. It is the CME activity provider's responsibility to submit participant completion information to ACCME for the purpose of granting ABIM MOC credit.

All other clinicians completing this activity will be issued a certificate of participation. To participate in this journal CME activity: (1) review the learning objectives and author disclosures; (2) study the education content; (3) take the posttest with a $75 \%$ minimum passing score and complete the evaluation at http://www.medscape.org/journal/eid; and (4) view/print certificate. For CME questions, see page 1036.

Release date: April 12, 2019; Expiration date: April 12, 2020

Learning Objectives

Upon completion of this activity, participants will be able to:

- $\quad$ Assess epidemiologic characteristics of Staphylococcus aureus bacteremia (SAB) cases in Denmark

- Distinguish age groups experiencing the biggest changes in the epidemiology of SAB

- $\quad$ Evaluate hospitalization data for SAB in Denmark

- $\quad$ Analyze mortality outcomes associated with SAB

\section{CME Editor}

Deborah Wenger, MBA, Copyeditor, Emerging Infectious Diseases. Disclosure: Deborah Wenger, MBA, has disclosed no relevant financial relationships.

\section{CME Author}

Charles P. Vega, MD, Health Sciences Clinical Professor of Family Medicine, University of California, Irvine School of Medicine, Irvine, California. Disclosure: Charles $P$. Vega, MD, has disclosed the following relevant financial relationships: served as an advisor or consultant for Johnson \& Johnson Pharmaceutical Research \& Development, L. L. C.; Genentech; GlaxoSmithKline; served as a speaker or a member of a speakers bureau for Shire.

\section{Authors}

Disclosures: Louise Thorlacius-Ussing, MD; Håkon Sandholdt, MSc; Anders Rhod Larsen, PhD; and Andreas Petersen, PhD, have disclosed no relevant financial relationships. Thomas Benfield, MD, DMSc, has disclosed the following relevant financial relationships: served as a speaker or a member of a speakers bureau for Boehringer Ingelheim Pharmaceuticals, Inc.; GlaxoSmithKline; Pfizer Inc.; received grants for clinical research from GlaxoSmithKline; Pfizer Inc.; owns stock, stock options, or bonds from ViroGates.

Author affiliations: Hvidovre Hospital, University of Copenhagen, Copenhagen, Denmark (L. Thorlacius-Ussing, H. Sandholdt, T. Benfield); Statens Serum Institut, Copenhagen (A. Larsen, A. Petersen)

DOI: https://doi.org/10.3201/eid2505.181733
Staphylococcus aureus bacteremia (SAB) is a major cause of illness and death worldwide. We analyzed temporal trends of SAB incidence and death in Denmark during 2008-2015. SAB incidence increased $48 \%$, from 20.76 to 30.37 per 100,000 person-years, during this period $(p<0.001)$. The largest change in incidence was observed for persons $\geq 80$ 
years of age: a $90 \%$ increase in the SAB rate $(p<0.001)$. After adjusting for demographic changes, annual rates increased $4.0 \%(95 \% \mathrm{Cl} 3.0-5.0)$ for persons $<80$ years of age, $8.4 \%(95 \% \mathrm{Cl} 7.0-11.0)$ for persons $80-89$ years of age, and $13.0 \%(95 \% \mathrm{Cl} 9.0-17.5)$ for persons $>90$ years of age. The 30-day case-fatality rate remained stable at $24 \%$; crude population death rates increased by $53 \%$ during 2008-2015 $(p<0.001)$. Specific causes and mechanisms for this rapid increase in $S A B$ incidence among the elderly population remain to be clarified.

Staphylococcus aureus is the most frequent gram-

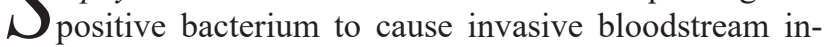
fection (1). S. aureus bacteremia (SAB) is associated with considerable illness and death, yielding a case-fatality rate of $20 \%-25 \%$ (2). The occurrence of SAB has changed over time (3-5). Increasing incidence rates of SAB have been reported worldwide throughout the past few decades $(3,5)$. However, more recent studies have reported stable or decreasing rates of $\mathrm{SAB}$, as well as improved short-term death rates $(1,2,4,6)$. As a result of demographic changes, including a rapidly increasing elderly population, contemporary analysis of the epidemiology of SAB is necessary to prioritize and allocate healthcare resources. Because concurrent conditions are more common with age, the changing demographics are of particular concern; older age and concurrent conditions are strongly associated with an increased risk for $\operatorname{SAB}(2,4,7)$.

Population-based studies are necessary to obtain valid epidemiologic data regarding SAB (8). Using surveillance data from the ongoing national registration of $\mathrm{SAB}$ in Denmark, we conducted a nationwide cohort study of temporal changes in SAB. The aims of this study were 2-fold. First, we analyzed temporal changes in SAB incidence; second, we assessed short-term death rates and associated risk factors.

\section{Methods}

\section{Study Setting}

We conducted a nationwide study of SAB in Denmark during 2008-2015. The population of Denmark comprised $5,475,791$ residents in 2008 and 5,659,715 residents in 2015; all had free access to tax-financed healthcare. This study was approved by the Danish Data Protection Agency (approval nos. 2009-41-4179 and 2014-41-3376). Legislation in Denmark does not require informed consent for register-based studies.

\section{Study Population}

We identified cases of SAB using data from the continuous national SAB surveillance in Denmark (5). Inclusion in the register was based on the identification of $S$. aureus in $\geq 1$ blood culture. We defined cases in the study as those in patients with a first-time episode of SAB recorded during January 1, 2008-December 31, 2015.

\section{Data Sources}

The unique civil registration number assigned to residents of Denmark by the Civil Registration System tracks information on vital and immigrant status and enables linkage of nationwide administrative healthcare registers on an individual level (9). The registry is updated daily.

The Danish National Patient Registry contains discharge diagnoses (from the International Classification of Diseases, 10th Revision) for residents regarding all hospital contacts (inpatient and outpatient) (10). The Danish National Bureau of Statistic provides data on the resident population and number of hospital admission and days in the hospital. The Danish Microbiology Database has conducted national surveillance on infectious diseases and microorganisms since 2010 (11).

\section{Variables of Interest}

We analyzed and stratified age by 11 age groups: $<1,1-9$; $10-19 ; 20-29 ; 30-39 ; 40-49 ; 50-59 ; 60-69 ; 70-79 ; 80-89$ and $\geq 90$ years. We used the Charlson Comorbidity Index (CCI) was used as a general measure of concurrent conditions; this index has previously been validated for $\mathrm{SAB}$ (12). We categorized CCI score into 3 levels: no concurrent conditions (CCI score $=0)$, intermediate $(\mathrm{CCI}$ score $=1-2)$, or high (CCI score $\geq 3)(2)$.

Recent hospital contact within 90 days before SAB was applied as a proxy of healthcare- and hospital-acquired SAB. We have previously validated this approach with a positive predictive value of $83 \%$ of distinguishing between healthcare/hospital- and community-associated SAB (13).

\section{Statistics}

We reported counts by median and interquartile range (IQR). We computed crude incidence rates of SAB overall and by age, sex, and calendar year and calculated the incidence rates as the number of SAB cases per 100,000 person-years at risk and estimated person-years at risk assuming a uniform death rate throughout the year in the background population. We assessed temporal trends by comparing the incidence rate ratio (IRR) in 3 time periods, 2008-2010, 2011-2012, and 2013-2015. In addition, we used a Poisson regression model to evaluate the association of calendar year and incidence of SAB and added all available variables to the model hypothesized as confounders for the outcome in question. We validated the model assumptions using a quasi-Poisson model.

We performed several sensitivity analyses to assess whether changes in SAB incidence were associated with the rate of $S$. aureus isolates per 10,000 blood cultures, 
per 100,000 hospital admissions, or per 100,000 hospital days; changes in the relative difference of CCI score during 2008-2014 between cases and the population controls, by randomly matching 10 population controls by age and gender to each $\mathrm{SAB}$ case; and the proportion of SAB cases during 2008-2014 with hospital contact within 90 days before the diagnosis of SAB. We reported all-cause death rates for $\mathrm{SAB}$ cases as a 30-day case-fatality rate (CFR) and deaths as a percentage of the number of cases. In addition, we calculated crude population death rates as deaths of SAB cases per 100,000 person-years in the background population and applied a mortality rate ratio (MRR) to assess temporal trends in death rates. We used logistic regression models to identify any association between risk factors and 30-day death rates and adjusted for the following covariates: age, gender, calendar year, CCI score, and 90-day prior hospital contact. We present risk estimates as odds ratio (OR) with $95 \%$ CIs, and for all analyses, we considered a $\mathrm{p}$ value $\leq 0.05$ to be significant. We performed statistical analyses using R software version 3.2.3 (R Project for Statistical Computing, https://www.r-project.org).

\section{Results}

\section{Demographics}

We identified a total of 11,054 incident cases of SAB during the study period. Demographics of the study population are provided in Table 1. In brief, the median age was 68 years (IQR 56-79 years); $62 \%$ of patients were male and 38\% female. More than $75 \%$ of SAB case-patients had $\geq 1$ concurrent condition recorded before the SAB episode. Methicillinresistant $S$. aureus (MRSA) accounted for $1.3 \%$ of cases.

\section{Incidence Rates}

The number of patients with SAB increased from 1,131 in 2008 to 1,731 in 2015 , corresponding to a $48 \%$ increase in incidence, from 20.76 (95\% CI 19.57-22.01) to 30.73 (95\% CI 29.30-32.21) (IRR 1.48 [95\% CI 1.37-1.59]), compared with an average annual incidence rate of 24.93 (95\% CI, 24.47-25.40) cases/100,000 person-yeras. The highest incidence rates were observed among male patients, the elderly, and infants $<1$ year of age (Table 2; Figure 1; Appendix Tables 1-3, http://wwwnc.cdc.gov/EID/ article/25/5/18-1733-App1.pdf).

For persons $\geq 80$ years of age, the incidence rate increased consistently throughout the 8-year study period (Figure 1). As such, during 2008-2015, the relative proportion of cases in persons $\geq 80$ years of age increased from $19.72 \%$ (95\% CI $17.40-22.04$ ) to $26.17 \%$ (95\% CI 24.10 28.24) of all cases. Further, the age-specific incidence rate increased significantly, by an estimated $56 \%$ (IRR 1.56 [95\% CI 1.41-1.72]) for persons 80-89 years of age and $92 \%$ (IRR1.92 [95\% CI 1.57-2.37]) for patients $\geq 90$ years of age in 2013-2015 compared with 2008-2010. For persons $<80$ years of age, only rates for patients $50-79$ years of age increased in 2013-2015 compared with 2008-2010 (50-59 years, IRR 1.24 [95\% CI 1.09-1.40]; 60-69 years, IRR 1.20 [95\% CI 1.10-1.32]; 70-79 years, IRR 1.26 [95\% CI 1.15-1.38]) (Appendix Table 1). For 2011-2012, the incidence rate for persons $<80$ years of age did not differ compared with the rates for 2008-2010. Gender did not affect the age-specific trends in SAB rate (Appendix Tables 2, 3).

Regression analysis indicated that age was strongly associated with SAB incidence (Table 2). Additionally, male case-patients had a 2-fold higher risk (IRR 2.00 [95\% CI 1.92-2.08]) of acquiring SAB than female case-patients. After adjustment for demographic changes, the estimated annual rates of SAB increased by 4\% (IRR 1.04 [95\% CI $1.03-1.05]$ ) for persons $<80$ years of age, $8 \%$ (IRR 1.08 [95\% CI 1.07-1.11]) for persons $80-89$ years of age, and $13 \%$ (IRR 1.13 [95\% CI 9.0-17.5]) for persons $\geq 90$ years (Figure 2; Appendix Figure 1).

\section{Blood Culture Activity, Hospital Admission, and Hospital Stays}

The number of blood cultures performed in the healthcare system in Denmark increased from 367,884 in 2010 to 480,892 in 2015 . The positivity rate of $S$. aureus per 10,000 blood cultures increased from 33.43 (95\% CI 31.59-35.36) to 36.00 (95\% CI 34.32-37.73) during that period, corresponding to an increase of $8 \%(95 \%$ CI $0 \%-16 \%)$ (Appendix Table 4). The rate of SAB cases per 10,000 blood

\begin{tabular}{lc}
\hline \multicolumn{2}{l}{ Table 1. Demographic characteristics of patients with } \\
\multicolumn{2}{l}{ Staphylococcus aureus bacteremia, Denmark, 2008-2015* } \\
\hline Characteristic & Value \\
\hline Sex & $4,161(37.6)$ \\
F & $6,893(62.4)$ \\
M & $282(2.6)$ \\
\hline Age, y & $157(1.4)$ \\
$<1$ & $208(1.9)$ \\
$1-9$ & $204(1.8)$ \\
$10-19$ & $374(3.4)$ \\
$20-29$ & $792(7.2)$ \\
$30-39$ & $1,326(12.0)$ \\
$40-49$ & $2,486(22.4)$ \\
$50-59$ & $2,561(23.2)$ \\
$60-69$ & $2,121(19.2)$ \\
$70-79$ & $543(4.9)$ \\
$80-89$ & $68(56.0-79.0)$ \\
$>90$ & $64(63.9-64.7)$ \\
Median (IQR) & \\
Mean (SD) & $2,599(23.5)$ \\
\hline CCI score & $4,171(37.7)$ \\
0 & $4,284(38.8)$ \\
$1-2$ & $10,911(98.7)$ \\
$>3$ & $143(1.3)$ \\
\hline MSSA & \\
MRSA &
\end{tabular}


Table 2. Incidence rate and incidence rate ratio of Staphylococcus aureus bacteremia stratified by sex and age, Denmark, 20082015*

\begin{tabular}{|c|c|c|c|}
\hline Characteristic & IR, SAB/100.000 PY $(95 \% \mathrm{Cl})$ & IRR $(95 \% \mathrm{Cl}) \dagger$ & $p$ value \\
\hline \multicolumn{4}{|l|}{ Sex } \\
\hline $\mathrm{F}$ & $18.62(18.06-19.19)$ & Referent & NA \\
\hline $\mathrm{M}$ & $31.35(30.61-32.10)$ & $2.00(1.92-2.08)$ & $<0.001$ \\
\hline \multicolumn{4}{|l|}{ Age, y } \\
\hline$<1$ & $57.96(51.28-65.12)$ & $17.37(14.28-21.13)$ & $<0.001$ \\
\hline $1-9$ & 3.35 (2.84-3.91) & Referent & NA \\
\hline $10-19$ & $3.75(3.26-4.30)$ & $1.12(0.91-1.38)$ & 0.281 \\
\hline $20-29$ & $3.84(3.33-4.41)$ & $1.15(0.91-1.38)$ & 0.199 \\
\hline 30-39 & $6.50(5.85-7.19)$ & $1.96(1.63-2.34)$ & $<0.001$ \\
\hline $40-49$ & $12.21(11.37-13.09)$ & 3.66 (3.09-4.34) & $<0.001$ \\
\hline $50-59$ & $22.92(21.70-24.18)$ & $6.88(5.84-8.12)$ & $<0.001$ \\
\hline $60-69$ & 46.14 (44.35-47.99) & $13.93(11.86-16.36)$ & $<0.001$ \\
\hline 70-79 & 81.26 (78.15-84.47) & $24.93(21.23-29.28)$ & $<0.001$ \\
\hline 80-89 & 145.17 (139.05-151.45) & 40.02 (33.35-48.03) & $<0.001$ \\
\hline$\geq 90$ & $197.03(180.80-214.32)$ & 49.97 (39.19-63.72) & $<0.001$ \\
\hline Period, per-year increment & NA & $1.04(1.03-1.05)$ & $<0.001$ \\
\hline
\end{tabular}

cultures remained stable for all age groups across the study period (Appendix Figure 2, Table 5). Annual hospital admissions increased from $1,175,452$ to $1,347,563$, but the total number of hospital days admitted decreased from $4,854,060$ to $4,067,222$ during 2008-2015. After adjustment, the number of SAB cases per 100,000 hospital admissions increased by $33 \%(95 \%$ CI $24 \%-44 \%)$ and $\mathrm{SAB}$ cases per 100,000 hospital days increased by $83 \%$ (95\% CI 69\%-97\%) (Appendix Table 4).

\section{Concurrent Conditions and Recent Hospital Contact before SAB}

Patients with SAB had significantly more concurrent conditions than the matched population controls (mean CCI 2.43 for case-patients compared with 0.81 for population controls; $\mathrm{p}<0.001)$. SAB case-patients and population controls both had increasingly more concurrent conditions during the study period, but the changes over time were similar for case-patients and population controls overall and stratified by age (Appendix Figure 3). Approximately 75\% (n = 6,707 ) of case-patients had had hospital contact within 90

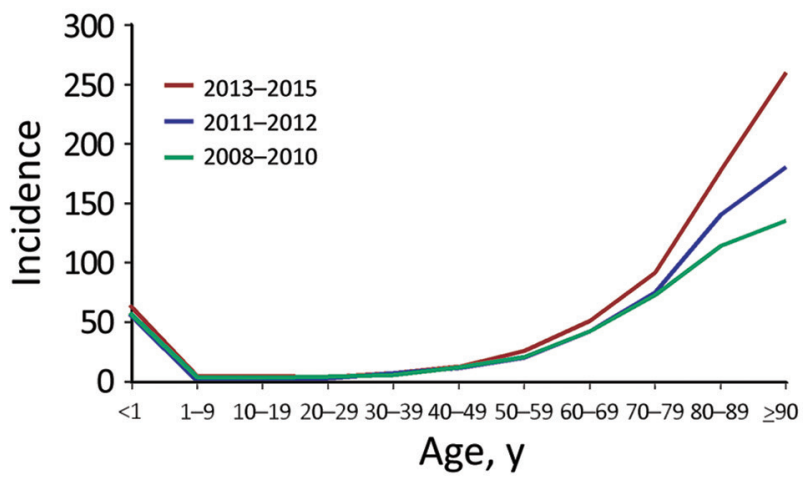

Figure 1. Temporal changes in Staphylococcus aureus bacteremia incidence (cases per 100,000 person-years), by age group and years, Denmark, 2008-2015. days before their SAB diagnosis. The proportion of SAB cases with recent hospital contact remained unchanged over the years (Appendix Figure 4, panel A). Stratification by age did not affect this result (Appendix Figure 4, panel B).

\section{CFR, Population Death Rate, and Associated Risk Factors}

In the 8-year study period, 30-day all-cause deaths increased from 271 in 2008 to 428 in 2015 . The population death rate for SAB rose significantly, from 4.97 (95\% CI $4.40-5.60)$ to 7.60 (95\% CI 6.90-8.35), corresponding to an estimated increase of 53\% (MRR 1.53 [95\% CI 1.311.78]) (Figure 3, panel A). Stratification by age group showed great variation in trends of the age-specific death rate, with the most rapid increase in death rate for the oldest age groups (Figure 3, panel B). For persons $<80$ years of age, the death rate increased by $25 \%$ (MRR 1.25 [95\% CI 1.03-1.51]).

The overall 30-day CFR for the study period was $24 \%$ (95\% CI 23\%-25\%) and remained unchanged over the years (Table 3; Figure 3, panel A). A higher 30-day CFR was observed with increasing age and CCI score, and for female compared with male case-patients (Table 3; Appendix Table 6). The 30-day CFR did not differ over time by gender or age (Appendix Figures 5,6). Multivariate analysis indicated that age was strongly associated with 30-day CFR (Table 3). Any concurrent condition before $\mathrm{SAB}$ was also associated with higher risk of death. Compared with persons with no concurrent conditions, the risk for death increased for persons with increasing CCI scores (OR 1.32 [95\% CI 1.14-1.55] for CCI score 1-2 and OR 1.67 [95\% CI 1.43-1.94] for CCI score $\geq 3$ ). Female sex was an independent risk factor for death compared with male sex (OR 1.20 [95\% CI 1.08-1.33]). In contrast, prior hospital contact and time period were not associated with 30-day CFR. 


\section{Discussion}

In this nationwide study, we evaluated trends of SAB incidence in the population of Denmark in an 8-year period, corresponding to $>40$ million patient-years. We report a $48 \%$ increase in SAB incidence during 2008-2015. Although the short-term death rate remained unchanged throughout the period, population-based death rates increased more than $50 \%$ because of the increase in SAB incidence.

The reported increase in SAB incidence was, in particular, a result of the major and consistent increase among persons $\geq 80$ years of age: the SAB rate among the oldest old ( $\geq 80$ years) rose with an alarming $90 \%$ during $2008-2015$, corresponding to an estimated annual increase of $8 \%-13 \%$ in incidence rate. In comparison, a $36 \%$ increase in the incidence rate was found for persons $<80$ years of age.

The differential increase in the SAB rate among age groups stresses the importance of comprehensive data that enable age-stratified analyses when investigating temporal trends in infectious diseases. Significant changes in death and incidence rates within subgroups of a population may otherwise not be apparent.

The increasing incidence rate observed in this study is contrary to most other recent studies. Stable or decreasing rates of SAB were reported in several large observational studies $(2,4-6,14,15)$. However, increasing rates of both community- and healthcare-acquired SAB were reported in a recent study in Finland (16). A higher occurrence of $\mathrm{SAB}$ is somewhat to be expected in an increasingly elderly population. However, our data showed an increase in SAB occurrence that exceeded contemporary changes in the population demographic profile.

Multiple factors may have influenced the changes in SAB incidence. Concurrent conditions form a strong risk factor associated with development of SAB; the increasing burden of concurrent conditions associated with aging could explain the higher SAB rate among the oldest elderly (2). The study population generally developed more concurrent conditions during the study period; thus, the number of persons at risk of acquiring SAB increased. However, because concurrent conditions increased similarly for

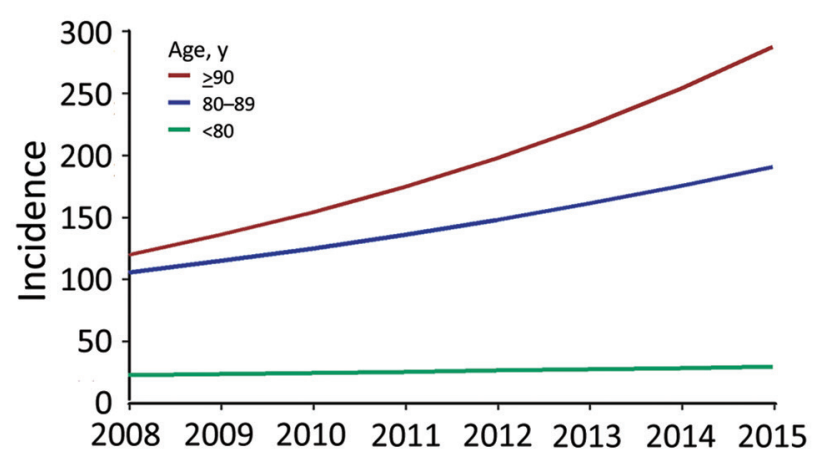

Figure 2. Increase in incidence of Staphylococcus aureus bacteremia for persons $>80$ years of age compared with younger persons, Denmark, 2008-2015.

SAB cases and population controls, the increasing incidence of SAB could not be explained solely by an increase in these conditions.

A more liberal use of invasive hospital procedures and immune modulating treatments may also contribute to a higher occurrence of SAB. Although the proportion of SAB cases with 90-day prior hospital contact remained unchanged throughout the study, we do not know whether rates of invasive procedures and immune-suppressive medication increased in the same period. Thus, rates of hospital contacts might not be an accurate measure; recent reports showed an increasing use of chemotherapy and invasive procedures over time, in particular among the elderly $(17,18)$.

The average life expectancy in Denmark increased during the study period, leading to an increasingly older and potentially more fragile population (19). There may be biologic risk factors of infectious diseases associated with aging that have not yet been identified. Physiologic agedependent changes, such as immunological senescence, are likely attributable, in part, to the higher vulnerability to infectious diseases among elderly persons $(20,21)$.

Higher rates of hospitalization and test activity and longer in-hospital stays may be other potential explanations for the increasing SAB rate (22). Blood culture activity
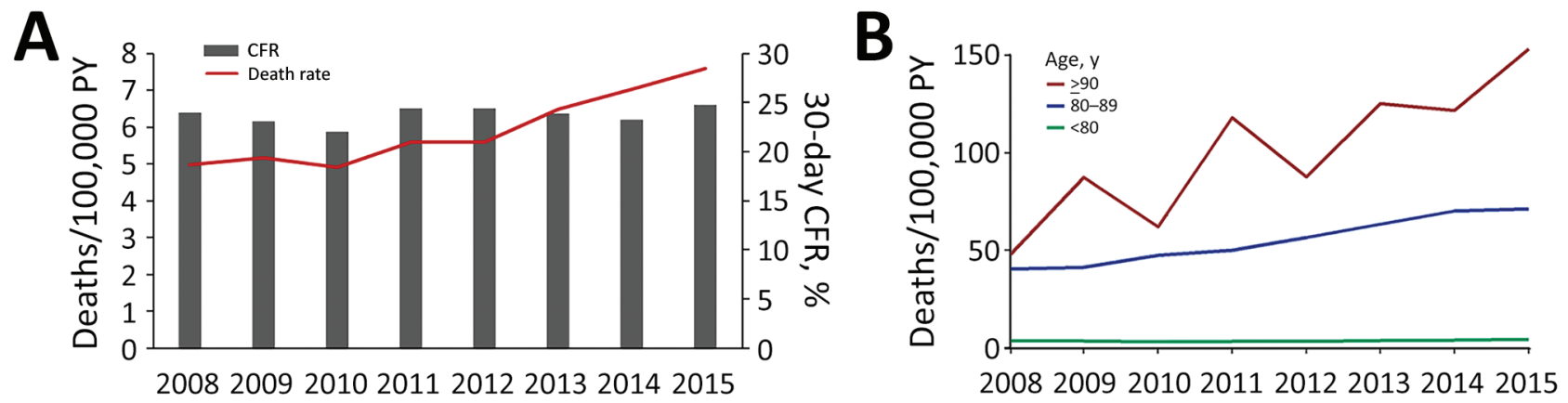

Figure 3. Staphylococcus aureus bacteremia deaths, Denmark, 2008-2015. A) Overall population death rate and 30-day CFR. B) Population death rates for persons $>80$ years of age compared with younger persons. PY, person-years. 
Table 3. CFR and associated risk of Staphylococcus aureus bacteremia, Denmark, 2008-2015*

\begin{tabular}{|c|c|c|c|}
\hline Characteristic & 30-d CFR $(95 \% \mathrm{Cl})$ & Multivariate OR (Cl 95\%) & $p$ value \\
\hline \multicolumn{4}{|c|}{ (x) } \\
\hline M & $21.88(20.79-23.01)$ & Referent & NA \\
\hline $\mathrm{F}$ & $26.92(25.36-28.54)$ & $1.20(1.08-1.33)$ & $<0.001$ \\
\hline \multicolumn{4}{|l|}{ Age, $y$} \\
\hline$<1$ & $6.74(4.06-10.52)$ & $4.68(1.05-20.85)$ & 0.043 \\
\hline$\overline{1}-9$ & $2.55(0.69-6.52)$ & Referent & NA \\
\hline $10-19$ & $1.92(0.52-4.92)$ & $1.71(0.31-9.50)$ & 0.538 \\
\hline $20-29$ & $2.94(1.08-6.40)$ & $2.22(0.44-11.20)$ & 0.333 \\
\hline 30-39 & $2.13(0.92-4.21)$ & $1.36(0.28-6.64)$ & 0.705 \\
\hline $40-49$ & $9.47(7.45-11.87)$ & $6.37(1.54-26.34)$ & 0.011 \\
\hline $50-59$ & 16.44 (14.33-18.77) & $10.79(2.64-44.06)$ & $<0.001$ \\
\hline $60-69$ & $19.79(18.08-21.62)$ & $13.00(3.19-52.89)$ & $<0.001$ \\
\hline $70-79$ & $27.80(25.80-29.92)$ & $19.69(4.85-79.94)$ & $<0.001$ \\
\hline $80-89$ & $38.10(35.51-40.82)$ & $32.26(7.94-131.03)$ & $<0.001$ \\
\hline$\geq 90$ & $51.93(46.05-58.36)$ & $58.48(14.26-239.82$ & $<0.001$ \\
\hline \multicolumn{4}{|l|}{$\mathrm{CCl}$ score } \\
\hline 0 & $14.20(12.79-15.72)$ & Referent & NA \\
\hline $1-2$ & $23.90(22.44-25.43)$ & $1.32(1.14-1.55)$ & $<0.001$ \\
\hline$\geq 3$ & $29.46(27.86-31.13)$ & $1.67(1.43-1.94)$ & $<0.001$ \\
\hline \multicolumn{4}{|l|}{ Hospital contact within $90 \mathrm{~d}$} \\
\hline No & $22.21(20.34-24.23)$ & Referent & NA \\
\hline Yes & 23.98 (22.82-25.18) & $1.10(0.97-1.24)$ & 0.130 \\
\hline Period, per-year increment & NA & $0.99(0.97-1.01)$ & 0.044 \\
\hline
\end{tabular}

increased by $31 \%$ during the study period but the increase in the $\mathrm{SAB}$ positivity rate was $8 \%$, suggesting that the increase in activity alone could not explain the observed changes. Further, a systematic increase in culture activity or higher sensitivity of culture systems would not explain a differential increase in incidence among age groups. Higher test rates would likely lead to the identification of milder cases of SAB. If so, a contemporary decline in the overall 30-day CFR would be expected, given higher survival rates among the less severe cases, but this was not the case.

Annual hospital admission rates increased by $15 \%$ and the number of days hospitalized declined by $16 \%$ during the study period, suggesting that changes in hospitalizations did not explain the increased rates of SAB. The increase in rates of $\mathrm{SAB}$ remained after adjustment for hospitalizations and hospital days. An increase in hospital-acquired infections is an unlikely explanation for the increased SAB rates because the proportion of $\mathrm{SAB}$ patients with and without 90-day prior hospital contact remained unchanged throughout the study.

A recent study found that the increase in sepsis rates and decline in death rates in California were associated with up-capture of less severely ill patients after introduction of guidance on coding based on the International Classification of Diseases, Ninth Revision (23). Our study, however, relied on blood culture positive cases of SAB; therefore, changes in coding practices are an unlikely explanation of our finding. Further, death rates were unchanged over time in our study.

In spite of a recent report of a decline in death associated with infectious diseases in general, SAB survival has not improved markedly during the past decades (24). The 30-day
CFR of $24 \%$ found in our study is in line with other reports (25-27). However, the population death rate rose 1.5-fold, to $7.60 / 100,000$ person-years by 2015 , because of the contemporary increase in SAB incidence. Thus, population death rates may represent a more accurate measure of the actual disease burden, as changes in incidence are reflected despite an unchanged 30-day CFR. Few studies have reported SAB death rates as population death rates for comparison and none have reported age-specific death rates $(28,29)$. Tom et al. reported a death rate from community-acquired $\mathrm{SAB}$ of $3.4 / 100,000$ person-years (28). A recent study from Norway on bloodstream infections found a death rate for $\mathrm{SAB}$ similar to ours, of 7/100,000 person-years (29).

A particular finding in our study was the close association between the changes in the overall death rate and the increase in the age-specific death rate for the oldest age groups. The higher incidence rates for the oldest age groups were directly reflected by higher population death rates. Death rates from SAB will likely increase even more over the next decades owing to the increasingly older population, which may also be the case for other invasive bloodstream infections. However, temporal population death rates from nonstaphylococcal bloodstream infections have not been reported; further studies are warranted to address this matter.

Contrary to the case with most bloodstream infections, female sex has been associated with an inferior outcome in SAB (25). In agreement with several previous observations, we found a significantly higher death rate among women compared with men $(7,25,30,31)$. The mechanisms underlying the observed gender differences in SAB death rates are not fully understood; a recent study did not find any gender-specific differences in clinical management, 
patient characteristics, or severity of the disease between men and women (32).

This study benefits from the large number of observational years, nationwide settings, and standardized registration. Nonetheless, some limitations must be noted. First, the increasing life expectancy during the study period could have led to an increasing median age within age groups and, consequently, an underestimation of the effect of age on incidence rates, in particular for the oldest persons. Second, the SAB definition was based solely on microbiological findings and not related to criteria of clinical infection such as systemic inflammatory response syndrome and sequential organ failure assessment score. Thus, we cannot preclude that a minor proportion of the positive blood cultures may be from contamination rather than clinical infection. Still, this possibility does not explain the age-dependent increase in SAB. Third, we were not able to stratify SAB origin by community-, healthcare-, and hospitalacquired infections, because these data were not accessible. Instead, we used a validated approach to access temporal changes in healthcare/hospital- and community-acquired infections. Fourth, data on blood culture activity were not available for 2008-2009; thus, we were not able to analyze trends in culture rates for the whole study period. Finally, we did not have access to clinical data regarding the primary focus of the infection, the severity of the disease, and the applied treatment strategies, as well as the effect of these potential risk factors on SAB death.

MRSA bacteremia is infrequent in Denmark $(1.3 \%$ of all SAB cases), which could theoretically limit the validity of the results of this study to settings with higher MRSA prevalence. Nevertheless, in most populations, methicillin-susceptible $S$. aureus (MSSA) bacteremia has remained prevalent despite the emergence of MRSA and, as such, several studies have concluded that MRSA bacteremia adds to the total burden of $\mathrm{SAB}$ rather than replacing MSSA bacteremia $(16,33)$. Thus, we believe that the observed changes in MSSA bacteremia in our study may be applicable to other populations.

In conclusion, SAB incidence in Denmark increased by $48 \%$ during $2008-2015$. SAB rates increased the most among the oldest age group, for whom the age-specific incidence rate nearly doubled and where increases in rates exceeded the contemporary changes in the population demographic profile. Furthermore, the short-term prognosis of SAB did not improve within the study period and, combined with the increasing incidence, population death rates rose significantly. Our results stress that infection prevention initiatives and improved care are warranted to reduce $\mathrm{SAB}$ incidence and improve outcomes. In addition, examinations of the burden of bloodstream infections caused by MSSA must be prioritized in future research; a specific focus should be on the frail elderly population.

\section{Acknowledgments}

The authors thank the departments of clinical microbiology throughout Denmark who contributed data to the national surveillance program.

\section{About the Author}

Dr. Thorlacius-Ussing is a physician and $\mathrm{PhD}$ student at Department of Infectious Diseases, Hvidovre Hospital, Copenhagen University, Denmark. Her primary research interests are epidemiology and management of invasive bloodstream infections.

\section{References}

1. Nielsen SL, Pedersen C, Jensen TG, Gradel KO, Kolmos HJ, Lassen AT. Decreasing incidence rates of bacteremia: a 9-year population-based study. J Infect. 2014;69:51-9. http://dx.doi.org/ 10.1016/j.jinf.2014.01.014

2. Mejer N, Westh H, Schønheyder HC, Jensen AG, Larsen AR, Skov R, et al.; Danish Staphylococcal Bacteraemia Study Group. Stable incidence and continued improvement in short term mortality of Staphylococcus aureus bacteraemia between 1995 and 2008. BMC Infect Dis. 2012;12:260. http://dx.doi.org/ 10.1186/1471-2334-12-260

3. Lyytikäinen O, Ruotsalainen E, Järvinen A, Valtonen V, Ruutu P. Trends and outcome of nosocomial and communityacquired bloodstream infections due to Staphylococcus aureus in Finland, 1995-2001. Eur J Clin Microbiol Infect Dis. 2005; 24:399-404. http://dx.doi.org/10.1007/s10096-005-1345-3

4. Laupland KB, Lyytikäinen O, Søgaard M, Kennedy KJ, Knudsen JD, Ostergaard C, et al.; International Bacteremia Surveillance Collaborative. The changing epidemiology of Staphylococcus aureus bloodstream infection: a multinational population-based surveillance study. Clin Microbiol Infect. 2013; 19:465-71. http://dx.doi.org/10.1111/j.1469-0691.2012.03903.x

5. Benfield T, Espersen F, Frimodt-Møller N, Jensen AG, Larsen AR, Pallesen LV, et al. Increasing incidence but decreasing in-hospital mortality of adult Staphylococcus aureus bacteraemia between 1981 and 2000. Clin Microbiol Infect. 2007;13:257-63. http://dx.doi.org/10.1111/j.1469-0691.2006.01589.x

6. Khatib R, Sharma M, Iyer S, Fakih MG, Obeid KM, Venugopal A, et al. Decreasing incidence of Staphylococcus aureus bacteremia over 9 years: greatest decline in community-associated methicillinsusceptible and hospital-acquired methicillin-resistant isolates. Am J Infect Control. 2013;41:210-3. http://dx.doi.org/10.1016/ j.ajic. 2012.03 .038

7. Braquet P, Alla F, Cornu C, Goehringer F, Piroth L, Chirouze C, et al.; VIRSTA-AEPEI study group. Factors associated with 12 week case-fatality in Staphylococcus aureus bacteraemia: a prospective cohort study. Clin Microbio 1 Infect. 2016;22:948.e1-7. http://dx.doi.org/10.1016/j.cmi.2016.07.034

8. Laupland KB. Incidence of bloodstream infection: a review of population-based studies. Clin Microbiol Infect. 2013;19:492-500. http://dx.doi.org/10.1111/1469-0691.12144

9. Schmidt M, Pedersen L, Sørensen HT. The Danish Civil Registration System as a tool in epidemiology. Eur J Epidemiol. 2014;29:541-9. http://dx.doi.org/10.1007/s10654-014-9930-3

10. Lynge E, Sandegaard JL, Rebolj M. The Danish National Patient Register. Scand J Public Health. 2011;39(supp17):30-3. http://dx.doi.org/10.1177/1403494811401482

11. Voldstedlund M, Haarh M, Mølbak K,; MiBa Board of Representatives. The Danish Microbiology Database (MiBa) 2010 to 2013. Euro Surveill. 2014;19:20667. http://dx.doi.org/ 10.2807/1560-7917.ES2014.19.1.20667 
12. Lesens O, Methlin C, Hansmann Y, Remy V, Martinot M, Bergin C, et al. Role of comorbidity in mortality related to Staphylococcus aureus bacteremia: a prospective study using the Charlson weighted index of comorbidity. Infect Control Hosp Epidemiol. 2003;24:890-6. http://dx.doi.org/10.1086/502156

13. Gotland N, Uhre ML, Mejer N, Skov R, Petersen A, Larsen AR, et al.; Danish Staphylococcal Bacteremia Study Group. Long-term mortality and causes of death associated with Staphylococcus aureus bacteremia. A matched cohort study. J Infect. 2016;73:34657. http://dx.doi.org/10.1016/j.jinf.2016.07.005

14. Mitchell BG, Collignon PJ, McCann R, Wilkinson IJ, Wells A. A major reduction in hospital-onset Staphylococcus aureus bacteremia in Australia - 12 years of progress: an observational study. Clin Infect Dis. 2014;59:969-75. http://dx.doi.org/10.1093/ cid/ciu508

15. David MZ, Daum RS, Bayer AS, Chambers HF, Fowler VG Jr, Miller LG, et al. Staphylococcus aureus bacteremia at 5 US academic medical centers, 2008-2011: significant geographic variation in community-onset infections. Clin Infect Dis. 2014; 59:798-807. http://dx.doi.org/10.1093/cid/ciu410

16. Jokinen E, Laine J, Huttunen R, Lyytikäinen O, Vuento R, Vuopio J, et al. Trends in incidence and resistance patterns of Staphylococcus aureus bacteremia. Infect Dis (Lond). 2018;50:528. http://dx.doi.org/10.1080/23744235.2017.1405276

17. van der Geest LGM, Haj Mohammad N, Besselink MGH, Lemmens VEPP, Portielje JEA, van Laarhoven HWM, et al.; Dutch Pancreatic Cancer Group. Nationwide trends in chemotherapy use and survival of elderly patients with metastatic pancreatic cancer. Cancer Med. 2017;6:2840-9. http://dx.doi.org/ 10.1002/cam4.1240

18. Pagé M, Doucet M, Eisenberg MJ, Behlouli H, Pilote L. Temporal trends in revascularization and outcomes after acute myocardial infarction among the very elderly. CMAJ. 2010;182:1415-20. http://dx.doi.org/10.1503/cmaj.092053

19. Statistics Denmark. Life expectancy [cited 2018 Oct 10]. https://www.dst.dk/en/Statistik/emner/befolkning-og-valg/ doedsfald-og-middellevetid/middellevetid

20. Gavazzi G, Krause K-H. Ageing and infection. Lancet Infect Dis. 2002;2:659-66. http://dx.doi.org/10.1016/S1473-3099(02)00437-1

21. Butcher S, Chahel H, Lord JM. Ageing and the neutrophil: no appetite for killing? Immunology. 2000;100:411-6. http://dx.doi.org/ 10.1046/j.1365-2567.2000.00079.x

22. Laupland KB, Niven DJ, Pasquill K, Parfitt EC, Steele L. Culturing rate and the surveillance of bloodstream infections: a populationbased assessment. Clin Microbiol Infect. 2018;24:910.e1-4. http://dx.doi.org/10.1016/j.cmi.2017.12.021

23. Gohil SK, Cao C, Phelan M, Tjoa T, Rhee C, Platt R, et al. Impact of policies on the rise in sepsis incidence, 2000-2010. Clin Infect Dis. 2016;62:695-703. http://dx.doi.org/10.1093/cid/civ1019

24. El Bcheraoui C, Mokdad AH, Dwyer-Lindgren L, Bertozzi-Villa A, Stubbs RW, Morozoff C, et al. Trends and patterns of differences in infectious disease mortality among US counties,
1980-2014. JAMA. 2018;319:1248-60. http://dx.doi.org/10.1001/ jama.2018.2089

25. Smit J, López-Cortés LE, Kaasch AJ, Søgaard M, Thomsen RW, Schønheyder HC, et al. Gender differences in the outcome of community-acquired Staphylococcus aureus bacteraemia: a historical population-based cohort study. Clin Microbiol Infect. 2017;23:27-32. http://dx.doi.org/10.1016/j.cmi.2016.06.002

26. Allard C, Carignan A, Bergevin M, Boulais I, Tremblay V, Robichaud P, et al. Secular changes in incidence and mortality associated with Staphylococcus aureus bacteraemia in Quebec, Canada, 1991-2005. Clin Microbiol Infect. 2008;14:421-8. http://dx.doi.org/10.1111/j.1469-0691.2008.01965.x

27. Søgaard M, Nørgaard M, Dethlefsen C, Schønheyder HC. Temporal changes in the incidence and 30-day mortality associated with bacteremia in hospitalized patients from 1992 through 2006: a population-based cohort study. Clin Infect Dis. 2011;52:61-9. http://dx.doi.org/10.1093/cid/ciq069

28. Tom S, Galbraith JC, Valiquette L, Jacobsson G, Collignon P, Schønheyder HC, et al.; International Bacteraemia Surveillance Collaborative. Case fatality ratio and mortality rate trends of community-onset Staphylococcus aureus bacteraemia. Clin Microbiol Infect. 2014;20:O630-2. http://dx.doi.org/10.1111/ 1469-0691.12564

29. Mehl A, Åsvold BO, Lydersen S, Paulsen J, Solligård E, Damås JK, et al. Burden of bloodstream infection in an area of mid-Norway 2002-2013: a prospective population-based observational study. BMC Infect Dis. 2017;17:205. http://dx.doi.org/10.1186/s12879-017-2291-2

30. Yahav D, Yassin S, Shaked H, Goldberg E, Bishara J, Paul M, et al. Risk factors for long-term mortality of Staphylococcus aureus bacteremia. Eur J Clin Microbiol Infect Dis. 2016;35:785-90. http://dx.doi.org/10.1007/s10096-016-2598-8

31. Mansur N, Hazzan R, Paul M, Bishara J, Leibovici L. Does sex affect 30-day mortality in Staphylococcus aureus bacteremia? Gend Med. 2012;9:463-70. http://dx.doi.org/10.1016/ j.genm.2012.10.009

32. Forsblom E, Kakriainen A, Ruotsalainen E, Järvinen A. Comparison of patient characteristics, clinical management, infectious specialist consultation, and outcome in men and women with methicillin-sensitive Staphylococcus aureus bacteremia: a propensity-score adjusted retrospective study. Infection. 2018;46:837-45. http://dx.doi.org/10.1007/s15010018-1216-3

33. Mostofsky E, Lipsitch M, Regev-Yochay G. Is methicillinresistant Staphylococcus aureus replacing methicillin-susceptible S. aureus? J Antimicrob Chemother. 2011;66:2199-214. http://dx.doi.org/10.1093/jac/dkr278

Address for correspondence: Louise Thorlacius-Ussing, Hvidovre Hospital Department of Infectious Diseases, Kettegaard Allé 30, 2650 Hvidovre, Denmark; email: louise.thorlacius-ussing@regionh.dk 